\title{
AN UPPER ESTIMATE OF INTEGRAL POINTS IN REAL SIMPLICES WITH AN APPLICATION TO SINGULARITY THEORY
}

\author{
Stephen T. Yau and Letian Zhang
}

\begin{abstract}
Let $\Delta\left(a_{1}, a_{2}, \cdots, a_{n}\right)$ be an $n$-dimensional real simplex with vertices at $\left(a_{1}, 0, \cdots, 0\right),\left(0, a_{2}, \cdots, 0\right), \cdots,\left(0,0, \cdots, a_{n}\right)$. Let $P_{\left(a_{1}, a_{2}, \cdots, a_{n}\right)}$ be the number of positive integral points lying in $\Delta\left(a_{1}, a_{2}, \cdots, a_{n}\right)$. In this paper we prove that $n ! P_{\left(a_{1}, a_{2}, \cdots, a_{n}\right)} \leq\left(a_{1}-1\right)\left(a_{2}-1\right) \cdots\left(a_{n}-1\right)$. As a consequence we have proved the Durfee conjecture for isolated weighted homogeneous singularities: $n ! p_{g} \leq \mu$, where $p_{g}$ and $\mu$ are the geometric genus and Milnor number of the singularity, respectively.
\end{abstract}

\section{Introduction}

Let $\Delta\left(a_{1}, a_{2}, \ldots, a_{n}\right)$ be an $n$-dimensional simplex described by

$$
\frac{x_{1}}{a_{1}}+\frac{x_{2}}{a_{2}}+\cdots+\frac{x_{n}}{a_{n}} \leq 1, \quad x_{1}, x_{2}, \ldots, x_{n} \geq 0,
$$

where $a_{1} \geq a_{2} \geq \cdots \geq a_{n} \geq 1$ are positive real numbers. Define $P_{\left(a_{1}, a_{2}, \ldots, a_{n}\right)}$ and $Q_{\left(a_{1}, a_{2}, \ldots, a_{n}\right)}$ to be the number of positive and nonnegative integral solutions of (1.1), respectively (i.e. the number of positive and nonnegative integral points in simplex $\left.\Delta\left(a_{1}, a_{2}, \ldots, a_{n}\right)\right)$. If we let $a=\frac{1}{a_{1}}+\frac{1}{a_{2}}+\cdots+\frac{1}{a_{n}}$, then $P_{\left(a_{1}, a_{2}, \ldots, a_{n}\right)}$ and $Q_{\left(a_{1}, a_{2}, \ldots, a_{n}\right)}$ are related by the following formulas:

$$
\begin{aligned}
Q_{\left(a_{1}, a_{2}, \ldots, a_{n}\right)} & =P_{\left(a_{1}(1+a), a_{2}(1+a), \ldots, a_{n}(1+a)\right)} \\
P_{\left(a_{1}, a_{2}, \ldots, a_{n}\right)} & =Q_{\left(a_{1}(1-a), a_{2}(1-a), \ldots, a_{n}(1-a)\right)} .
\end{aligned}
$$

Hence, the study of $P_{\left(a_{1}, a_{2}, \ldots, a_{n}\right)}$ and the study of $Q_{\left(a_{1}, a_{2}, \ldots, a_{n}\right)}$ are equivalent. The computation of $Q_{\left(a_{1}, a_{2}, \ldots, a_{n}\right)}$ has received attention from many distinguished mathematicians. Hardy and Littlewood wrote several papers on the subject that have applications to problems of Diophantine approximation ([Ha-Li 1], [Ha-Li 2], [HaLi 3]). D. C. Spencer followed up the efforts of Hardy and Littlewood and wrote two papers on the estimation of $Q_{\left(a_{1}, a_{2}, \ldots, a_{n}\right)}$ as well ([Sp 1], [Sp 2]). Their results, however, are asymptotic in nature and are not useful in the applications described below. In recent years, tremendous effort has been put into finding exact formulas for $Q_{\left(a_{1}, a_{2}, \ldots, a_{n}\right)}$ and $P_{\left(a_{1}, a_{2}, \ldots, a_{n}\right)}$ where $a_{1}, a_{2}, \ldots, a_{n}$ are positive integers (see [Mo], $[\mathrm{Po}],[\mathrm{Ca}-\mathrm{Sh}],[\mathrm{Br}-\mathrm{Ve}],[\mathrm{Di}-\mathrm{Ro}],[\mathrm{Ka}-\mathrm{Kh}])$. However, since these results are limited to integral simplices, they have no known application to number theory. Furthermore, the exact formulas involve generalized Dedekind sums or other complicated terms

Received by the editors August 4, 2005.

First author is a Ze Jiang Professor at East China Normal University and his research is partially supported by the NSA. 
[Ba], and therefore it is difficult to determine the order of magnitude of $P_{\left(a_{1}, a_{2}, \ldots, a_{n}\right)}$. Ideally, we would like to get a formula for $P_{\left(a_{1}, a_{2}, \ldots, a_{n}\right)}$ in terms of a polynomial in $a_{1}, a_{2}, \ldots, a_{n}$, where $a_{1}, a_{2}, \ldots, a_{n}$ are not limited to integers, but can be any positive real numbers. Although such an exact formula may not exist, a relatively sharp upper estimate would suffice for the purpose of many applications in number theory and singularity theory. Barvinok and Pommersheim [Ba-Po] wrote an excellent article on topics related to lattice points in rational polyhedra. Currently the research area of lattice points in simplices is extremely active. For more information, we refer the readers to the collection "Integer Points in Polyhedra - Geometry, Number Theory, Algebra, Optimization," a Snowbird Conference Proceedings recently published by the AMS (Contemporary Mathematics, vol. 374, 2005).

According to Granville [Gr], finding an upper polynomial estimate of $P_{\left(a_{1}, a_{2}, \ldots, a_{n}\right)}$ is an extremely important subject in number theory. It could be applied to finding large gaps between primes, to Waring's problem, to primality testing and factoring algorithms, and to bounds for the least prime $k$-th power residues and non-residues $(\bmod n)$. Given a set $\mathcal{P}$ of primes $p_{1}<p_{2}<\cdots<p_{n}<y$, number theorists are interested in counting the number of integers $m \leq y^{u}$ where $m=p_{1}^{l_{1}} p_{2}^{l_{2}} \cdots p_{n}^{l_{n}}$ for all $u \geq 2$. This is equivalent to counting the number of $\left(l_{1}, l_{2}, \ldots, l_{n}\right) \in \mathbb{Z}_{\geq 0}^{n}$ such that $l_{1} p_{1}+l_{2} p_{2}+\cdots+l_{n} p_{n} \leq \log y^{u}$, which is also equivalent to counting the number of $\left(l_{1}, l_{2}, \ldots, l_{n}\right) \in \mathbb{Z}_{\geq 0}^{n}$ such that

$$
\frac{l_{1}}{a_{1}}+\frac{l_{2}}{a_{2}}+\cdots+\frac{l_{n}}{a_{n}} \leq 1, \text { where } a_{i}=\frac{\log y^{u}}{\log p_{i}} .
$$

Observe that the $a_{i}$ 's are not integral in general. For more information about applications of $P_{\left(a_{1}, a_{2}, \ldots, a_{n}\right)}$ and $Q_{\left(a_{1}, a_{2}, \ldots, a_{n}\right)}$, see Carl Pomerance's ICM 1994 lecture at Zürich [Pom 1] and his lecture notes [Pom 2].

The current method for counting $P_{\left(a_{1}, a_{2}, \ldots, a_{n}\right)}$ is the polynomial estimate (1.6) provided by number theorists. Attach a unit cube to the right of and above each lattice point of $\Delta\left(a_{1}, a_{2}, \ldots, a_{n}\right)$. Then

$$
\begin{aligned}
Q_{\left(a_{1}, a_{2}, \ldots, a_{n}\right)} & =\sum \text { volume of the unit cube attached to each lattice point } \\
& \leq \text { volume of }\left\{\left(x_{1}, x_{2}, \ldots, x_{n}\right) \in \mathbb{R}_{+}^{n}: \sum_{i=1}^{n} \frac{x_{1}-1}{a_{i}} \leq 1\right\} \\
& =\frac{1}{n !}\left(a_{1} a_{2} \cdots a_{n}\right)\left(1+\sum_{i=1}^{n} \frac{1}{a_{i}}\right)^{n} .
\end{aligned}
$$

In view of (1.2), (1.5) can be rewritten as

$$
P_{\left(a_{1}, a_{2}, \ldots, a_{n}\right)} \leq \frac{1}{n !} a_{1} a_{2} \cdots a_{n} .
$$

The estimate of $P_{\left(a_{1}, a_{2}, \ldots, a_{n}\right)}$ given by (1.6) is interesting. However, it is not strong enough to be useful, particularly when many of the $a_{i}$ 's are small $[\mathrm{Gr}]$. The purpose of this paper is to prove the following upper bound. 
Theorem 1.1. Let $P_{n}=P_{\left(a_{1}, a_{2}, \ldots, a_{n}\right)}=\#\left\{\left(x_{1}, \ldots, x_{n}\right) \in \mathbb{Z}_{+}^{n}: \frac{x_{1}}{a_{1}}+\frac{x_{2}}{a_{2}}+\cdots+\frac{x_{n}}{a_{n}} \leq\right.$ $1\}$, where $a_{1} \geq a_{2} \geq \cdots \geq a_{n} \geq 1$ are real numbers. If $n \geq 3$, then

$$
n ! \cdot P_{n} \leq\left(a_{1}-1\right)\left(a_{2}-1\right) \cdots\left(a_{n}-1\right) .
$$

Equality in (1.7) holds if and only if $a_{n}=1$.

Several mathematicians have attempted to prove Theorem 1.1 for separate cases of $n$. In fact, Theorem 1.1 was proven for $n=3$ by $\mathrm{Xu}$ and $\mathrm{Yau}[\mathrm{Xu}-\mathrm{Ya} 1]$, for $n=4$ and 5 by $\mathrm{Xu}$, Lin and $\mathrm{Yau}[\mathrm{Xu}-\mathrm{Ya} 2]$ [Li-Ya 1] [Li-Ya 2], and for $n=6$ by Wang and Yau $[\mathrm{Wa}-\mathrm{Ya}]$.

In geometry and in singularity theory, Theorem 1.1 is connected with the Durfee conjecture. Let $f:\left(\mathbb{C}^{n}, 0\right) \rightarrow(\mathbb{C}, 0)$ be a germ of a complex analytic function with an isolated critical point at the origin, and let $M$ be a resolution of $V=\left\{\left(z_{1}, z_{2}, \ldots, z_{n}\right) \in\right.$ $\left.\mathbb{C}^{n}: f\left(z_{1}, z_{2}, \ldots, z_{n}\right)=0\right\}$. The Milnor number of the singularity $(V, 0)$ is

$$
\mu=\operatorname{dim} \mathbb{C}\left\{z_{1}, z_{2}, \ldots, z_{n}\right\} /\left(f_{z_{1}}, f_{z_{2}}, \ldots, f_{z_{n}}\right) .
$$

The geometric genus of the singularity $(V, 0)$ is

$$
p_{g}=\operatorname{dim} H^{n-2}(M, \mathcal{O}) .
$$

Both $\mu$ and $p_{g}$ are important invariants of the singularity $(V, 0)$. As a corollary of Theorem 1.1, we have proven the following Durfee conjecture [Du] asked in 1978.

Theorem 1.2 (Durfee conjecture). Let $(V, 0)$ be an isolated singularity defined by a weighted homogeneous polynomial $f\left(z_{1}, z_{2}, \ldots, z_{n}\right)$. Then $n ! \cdot p_{g} \leq \mu$ and equality holds if and only if $\mu=0$.

The importance of the Durfee conjecture is that it gives a necessary condition for a singularity to be a hypersurface. It also gives an obstruction to embedding a strongly pseudo-convex $(2 n-1)$-dimensional $C R$-manifold in $\mathbb{C}^{n+1}$.

The connection between the Durfee conjecture and the upper polynomial estimate of $P_{\left(a_{1}, a_{2}, \ldots, a_{n}\right)}$ in real simplices is as follows. A polynomial $f\left(z_{1}, z_{2}, \ldots, z_{n}\right)$ is weighted homogeneous of the type $\left(w_{1}, w_{2}, \ldots, w_{n}\right)$, where $w_{1}, w_{2}, \ldots, w_{n}$ are fixed positive rational numbers, if $f$ can be expressed as a linear combination of monomials $z_{1}^{i_{1}} z_{2}^{i_{2}} \cdots z_{n}^{i_{n}}$ for which $\frac{i_{1}}{w_{1}}+\frac{i_{2}}{w_{2}}+\cdots+\frac{i_{n}}{w_{n}}=1$. If $f\left(z_{1}, z_{2}, \ldots, z_{n}\right)$ is a weighted homogeneous polynomial of type $\left(a_{1}, a_{2}, \ldots, a_{n}\right)$ with an isolated singularity at the origin, then Milnor and Orlik [Mi-Or] have proven that $\mu=\left(a_{1}-1\right)\left(a_{2}-1\right) \cdots\left(a_{n}-1\right)$. On the other hand, Merle and Teissier [Me-Te] showed that $p_{g}$ is exactly the number $P_{n}$ of positive integral points appearing in Theorem 1.1 (the Rough Estimate GLY conjecture). Therefore by proving Theorem 1.1, we have proven the Durfee conjecture.

Theorem 1.1 is the Rough Upper Estimate in the GLY conjecture [ $\mathrm{Li}-\mathrm{Ya} 3]$ [WaYa]. The Durfee conjecture in Theorem 1.2 is not sharp although it has been an open question for more than a quarter of century. In 1995, the first author formulated the Yau conjecture (see Section 2 below), which is sharper than the Durfee conjecture. More importantly, it gives an intrinsic characterization of homogeneous singularities. In order to prove the Yau conjecture, Lin and Yau [Li-Ya 3] [Wa-Ya], independently Granville, formulated the Sharp Upper Estimate GLY conjecture (see Section 2 below). The Sharp Estimate GLY conjecture is true only if $a_{n}$ is sufficiently large. Consequently, when we use induction to prove the Sharp Estimate by 
slicing an $n$-dimensional simplex along the $x_{n}$-axis into several $(n-1)$-dimensional simplices, we cannot apply the lower-dimensional Sharp Estimate conjecture to every level. The Rough Estimate is necessary in order to complete the proof of the Sharp Estimate. Hence, proving the Rough Estimate GLY conjecture is a critical first step to proving the complete GLY conjecture. We hope to address the Sharp Estimate GLY conjecture in the future paper.

\section{The GLY conjecture on number of integral points and the Yau conjecture in singularity theory}

The following is a thirty-year-old problem in singularity theory.

Problem: Let $f:\left(\mathbb{C}^{n}, 0\right) \rightarrow(\mathbb{C}, 0)$ be a complex analytic function with isolated critical point at the origin. Find an intrinsic characterization for $f$ to be a homogeneous polynomial.

In 1971, Saito [Sa] gave an intrinsic characterization for $f$ to be a weighted homogeneous polynomial.

Theorem 2.1 (Saito $[\mathrm{Sa}])$. Let $f:\left(\mathbb{C}^{n}, 0\right) \rightarrow(\mathbb{C}, 0)$ be a complex analytic function with isolated critical point at the origin. Then $f$ is a weighted homogeneous polynomial after biholomorphic change of coordinates if and only if $\mu=\tau$, where

$\mu=\operatorname{dim} \mathbb{C}\left\{z_{1}, z_{2}, \cdots, z_{n}\right\} /\left(f_{z_{1}}, f_{z_{2}}, \cdots, f_{z_{n}}\right)$ and

$\tau=\operatorname{dim} \mathbb{C}\left\{z_{1}, z_{2}, \cdots, z_{n}\right\} /\left(f, f_{z_{1}}, f_{z_{2}}, \cdots, f_{z_{n}}\right)$.

In order to characterize homogeneous polynomials with isolated singularity, the first author made the following conjecture in 1995.

Conjecture 2.1 (Yau Conjecture). Let $f:\left(\mathbb{C}^{n}, 0\right) \rightarrow(\mathbb{C}, 0)$ be a weighted homogeneous polynomial with an isolated singularity at the origin. Let $\mu, p_{g}$, and $\nu$ be the Milnor number, geometric genus and multiplicity of the singularity $V=\{z: f(z)=0\}$, then

$$
\mu-p(\nu) \geq n ! p_{g}
$$

where $p(\nu)=(\nu-1)^{n}-\nu(\nu-1) \cdots(\nu-n+1)$, and equality holds if and only if $f$ is a homogeneous polynomial.

Theorem 2.1 together with Yau conjecture will give an intrinsic characterization for a complex analytic function to be a homogeneous function after a biholomorphic change of variables. The Yau conjecture was answered affirmatively by Xu and Yau $[\mathrm{Xu}-\mathrm{Ya} 2]$ for $n=3$ and Lin and Yau [Li-Ya 4] for $n=4$. In order to prove the Yau conjecture above, Lin, Yau [Li-Ya 3], and Granville have formulated the following GLY conjecture.

Before we state the GLY conjecture, it is convenient to introduce some notations. Recall the Stirling number of the first kind (see $[\mathrm{Co}]$ for more information on Stirling number):

$$
s_{k}^{n-1}=\sum_{1 \leq i_{1}<i_{2}<\cdots<i_{k} \leq n-1} i_{1} i_{2} \cdots i_{k}, S_{0}^{n-1}=1, S_{n-1}^{n-1}=1 \cdot 2 \cdots(n-1),
$$


where $i_{1}, i_{2}, \cdots, i_{k}$ are integers. It has the following property:

$$
\begin{aligned}
& x(x-1)(x-2) \cdots(x-n+1) \\
= & x^{n}-\left(\sum_{i=1}^{n} i_{1}\right) x^{n-1}+(-1)^{2}\left(\sum_{1 \leq i_{1}<i_{2} \leq n-1} i_{1} i_{2}\right) x^{n-2} \\
& +\cdots+(-1)^{k}\left(\sum_{1 \leq i_{1}<i_{2}<\cdots<i_{k} \leq n-1} i_{1} i_{2} \cdots i_{k}\right) x^{n-k}+\cdots+(-1)^{n-1}\left(\prod_{i=1}^{n-1} i\right) x \\
= & x^{n}+(-1) S_{1}^{n-1} x^{n-1}+(-1)^{2} S_{2}^{n-1} x^{n-2}+\cdots+(-1)^{k} S_{k}^{n-1} x^{n-k} \\
& +\cdots+(-1)^{n-1} S_{n-1}^{n-1} x .
\end{aligned}
$$

Let $a_{1}, a_{2}, \cdots, a_{n}$ be positive real numbers. We shall denote

$$
\begin{aligned}
A_{n-k}^{n} & =\left(\prod_{i=1}^{n} a_{i}\right) \sum_{1 \leq i_{1}<i_{2}<\cdots<i_{k} \leq n} \frac{1}{a_{i_{1}} a_{i_{2}} \cdots a_{i_{k}}}, \\
A_{n}^{n} & =\prod_{i=1}^{n} a_{i}, \quad A_{0}^{n}=1 .
\end{aligned}
$$

Observe that $A_{n-k}^{n}$ is a polynomial in $a_{1}, a_{2}, \cdots, a_{n}$ of degree $n-k$.

Conjecture 2.2 (Granville-Lin-Yau (GLY) conjecture [Li-Ya 3] [Wa-Ya]). Let $P_{n}=$ $P_{\left(a_{1}, a_{2}, \ldots, a_{n}\right)}=\#\left\{\left(x_{1}, \ldots, x_{n}\right) \in \mathbb{Z}_{+}^{n}: \frac{x_{1}}{a_{1}}+\frac{x_{2}}{a_{2}}+\cdots+\frac{x_{n}}{a_{n}} \leq 1\right\}$, where $a_{1} \geq a_{2} \geq$ $\cdots \geq a_{n} \geq 1$ are real numbers. If $n \geq 3$, then

(I) Rough (General) Upper Estimate For all $a_{n} \geq 1$,

$$
n ! \cdot P_{n} \leq\left(a_{1}-1\right)\left(a_{2}-1\right) \cdots\left(a_{n}-1\right) .
$$

Equality holds if and only if $a_{n}=1$.

(II) Sharp Upper Estimate For $a_{n}$ sufficiently large: there exists an integer $\beta_{n}(n)$ that depends on $n$ such that when $a_{n} \geq \beta_{n}(n)$, then

$$
\begin{aligned}
n ! \cdot P_{\left(a_{1}, \ldots, a_{n}\right) \leq} \leq & A_{n}^{n}+(-1) \frac{S_{1}^{n-1}}{n} A_{n-1}^{n}+(-1)^{2} \frac{S_{2}^{n-1}}{\left(\begin{array}{c}
n-1 \\
1
\end{array}\right)} A_{n-2}^{n-1}+(-1)^{3} \frac{S_{3}^{n-1}}{\left(\begin{array}{c}
n-1 \\
2
\end{array}\right)} A_{n-3}^{n-1} \\
& +\cdots+(-1)^{k+1} \frac{S_{k+1}^{n-1}}{\left(\begin{array}{c}
n-1 \\
k
\end{array}\right)} A_{n-k-1}^{n-1}+\cdots+(-1)^{n-1} \frac{S_{n-1}^{n-1}}{\left(\begin{array}{c}
n-1 \\
n-2
\end{array}\right)} A_{1}^{n-1} .
\end{aligned}
$$

Equality holds if and only if $a_{1}=a_{2}=\cdots=a_{n} \in \mathbb{Z}_{\geq 0}$.

The GLY conjecture was proven by Xu and Yau for $n=3[\mathrm{Xu}-\mathrm{Ya} 1]$ and $n=4$ $[\mathrm{Xu}-\mathrm{Ya} 3]$, Lin and Yau for $n=5[\mathrm{Li}-\mathrm{Ya} 2]$ and Wang and Yau for $3 \leq n \leq 6$ [Wa-Ya]. In fact, Wang and Yau's method in [Wa-Ya] can be used to prove the GLY conjecture for any fixed $n$. It has been checked that the GLY conjecture is true for $n \leq 10$. However, it takes a long time (several weeks for $n=10$ ) for computer to do the computation. The purpose of this paper is to give a proof of the Rough Estimate GLY conjecture for all $n$. 


\section{Three lemmas}

Before proving Theorem 1.1, we need to establish three technical lemmas.

Lemma 3.1. Given any positive real number $\beta$ where $0<\beta<1$, let $a>1$ be any number such that $\beta=a-\lfloor a\rfloor$, where $\lfloor a\rfloor$ denotes the greatest positive integer less than or equal to $a$. If $n \geq 3$, then

$$
a-1>(n+1) \sum_{k=0}^{\lfloor a\rfloor-1} \frac{(k+\beta)^{n}}{a^{n}} .
$$

Proof. We shall prove (3.1) by induction on $\lfloor a\rfloor$. Consider the expression

$$
a-1-(n+1) \sum_{k=0}^{\lfloor a\rfloor-1} \frac{(k+\beta)^{n}}{a^{n}} .
$$

For $\lfloor a\rfloor=1$, we have $a=1+\beta$ and $\lfloor a\rfloor-1=0$, therefore (3.2) becomes

$$
\begin{aligned}
a-1-(n+1) \sum_{k=0}^{\lfloor a\rfloor-1} \frac{(k+\beta)^{n}}{a^{n}} & =1+\beta-1-(n+1) \frac{\beta^{n}}{(1+\beta)^{n}} \\
& =\beta\left[1-\frac{n \beta^{n-1}+\beta^{n-1}}{(1+\beta)^{n}}\right] .
\end{aligned}
$$

For $n \geq 2,(1+\beta)^{n}=\beta^{n}+n \beta^{n-1}+\cdots+1>n \beta^{n-1}+\beta^{n-1}$, so the right-hand-side of (3.3) is positive. To finish the proof, we need to show that if the statement of Lemma 3.1 is true for $a$, then it is also true for $a$. By the induction hypothesis, we have

$$
a-1>(n+1) \sum_{k=0}^{\lfloor a\rfloor-1} \frac{(k+\beta)^{n}}{a^{n}}
$$

Then

$$
\begin{aligned}
(a+1)-1-(n+1) \sum_{k=0}^{\lfloor a+1\rfloor-1} \frac{(k+\beta)^{n}}{(a+1)^{n}} & =a-\frac{n+1}{(a+1)^{n}}\left(\sum_{k=0}^{\lfloor a\rfloor-1}(k+\beta)^{n}+a^{n}\right) \\
& >a-\frac{n+1}{(a+1)^{n}}\left(\frac{a^{n}(a-1)}{n+1}+a^{n}\right) \\
& =a\left[1-\frac{a^{n}+n a^{n-1}}{(a+1)^{n}}\right] \\
& >0 .
\end{aligned}
$$

The last inequality in (3.5) comes from $(a+1)^{n}=a^{n}+n a^{n-1}+\cdots+1>a^{n}+n a^{n-1}$.

Lemma 3.2. Let $m \geq 2$ and $n \geq 3$ be positive integers, then

$$
m-1>(n+1) \sum_{k=1}^{m-1} \frac{k^{n}}{m^{n}} .
$$

Proof. It is easy to see that Lemma 3.2 is true for $m=2$. The proof of general $m \geq 2$ follows easily by induction. The argument is identical to that of Lemma 3.1. 
Proposition 3.1. Given any positive real number $\beta$ where $0 \leq \beta<1$, let $a>1$ be any number such that $\beta=a-\lfloor a\rfloor$, where $\lfloor a\rfloor$ denotes the greatest positive integer less than or equal to $a$. If $n \geq 3$, then

$$
a-1>(n+1) \sum_{k=0}^{\lfloor a\rfloor-1} \frac{(k+\beta)^{n}}{a^{n}} .
$$

Proof. Immediate consequence of Lemma 3.1 and Lemma 3.2.

Lemma 3.3. Let $a_{j-1}, a_{j}, \ldots, a_{n+1}$ be real numbers and $\beta=a_{n+1}-\left\lfloor a_{n+1}\right\rfloor$. Assume that $a_{j-1}>1$ and $a_{j} \geq a_{j+1} \geq \cdots \geq a_{n} \geq a_{n+1}>1$. If $\frac{a_{n}}{a_{n+1}} \beta \geq 1$, and

$$
\prod_{i=j}^{n+1}\left(a_{i}-1\right)>(n+1) \sum_{k=0}^{\left\lfloor a_{n+1}\right\rfloor-1}\left[\frac{(k+\beta)^{j-1}}{a_{n+1}^{j-1}} \prod_{i=j}^{n}\left(\frac{a_{i}}{a_{n+1}}(k+\beta)-1\right)\right],
$$

then

$$
\prod_{i=j-1}^{n+1}\left(a_{i}-1\right)>(n+1) \sum_{k=0}^{\left\lfloor a_{n+1}\right\rfloor-1}\left[\frac{(k+\beta)^{j-2}}{a_{n+1}^{j-2}} \prod_{i=j-1}^{n}\left(\frac{a_{i}}{a_{n+1}}(k+\beta)-1\right)\right] .
$$

Proof. For fixed $a_{j} \geq a_{j+1} \geq \cdots \geq a_{n} \geq a_{n+1}>1$, let

$$
F\left(a_{j-1}\right)=\prod_{i=j-1}^{n+1}\left(a_{i}-1\right)-(n+1) \sum_{k=0}^{\left\lfloor a_{n+1}\right\rfloor-1}\left[\frac{(k+\beta)^{j-2}}{a_{n+1}^{j-2}} \prod_{i=j-1}^{n}\left(\frac{a_{i}}{a_{n+1}}(k+\beta)-1\right)\right] .
$$

To prove the lemma, we only need to prove that $F\left(a_{j-1}\right)$ is a strictly increasing function of $a_{j-1}$ and $F(1) \geq 0$.

If $a_{j-1}=1$, then $\frac{a_{j-1}}{a_{n+1}}(k+\beta)-1<0$ for $0 \leq k \leq\left\lfloor a_{n+1}\right\rfloor-1$. Moreover, by the assumptions $a_{j} \geq a_{j+1} \geq \cdots \geq a_{n} \geq a_{n+1}>1$ and $\frac{a_{n}}{a_{n+1}} \beta \geq 1$, we have $\frac{a_{i}}{a_{n+1}} \beta \geq 1$ for all $i$ where $j \leq i \leq n$. Therefore

$$
\prod_{i=j}^{n}\left(\frac{a_{i}}{a_{n+1}}(k+\beta)-1\right) \geq 0 \text { for } 0 \leq k \leq\left\lfloor a_{n+1}\right\rfloor-1,
$$

and

$$
(n+1) \sum_{k=0}^{\left\lfloor a_{n+1}\right\rfloor-1}\left[\frac{(k+\beta)^{j-2}}{a_{n+1}^{j-2}} \prod_{i=j-1}^{n}\left(\frac{a_{i}}{a_{n+1}}(k+\beta)-1\right)\right] \leq 0 .
$$

Hence, we have shown that $F(1) \geq 0$. Next, we compute

$$
\begin{aligned}
\frac{d F}{d a_{j-1}} & =\prod_{i=j}^{n+1}\left(a_{i}-1\right)-(n+1) \sum_{k=0}^{\left\lfloor a_{n+1}\right\rfloor-1}\left[\frac{(k+\beta)^{j-1}}{a_{n+1}^{j-1}} \prod_{i=j}^{n}\left(\frac{a_{i}}{a_{n+1}}(k+\beta)-1\right)\right] \\
& >0 \text { by (3.8), }
\end{aligned}
$$

so $F\left(a_{j-1}\right)$ is a strictly increasing function of $a_{j-1}$. Thus, $F\left(a_{j-1}\right)>0$ for $a_{j-1}>$ 1 . 
Now we introduce Lemma 3.4, which is slightly different from Lemma 3.3. In Lemma 3.4, we let $\frac{a_{n}}{a_{n+1}} \beta<1$ and ignore the layer $k=0$ on the right-hand-side of $(3.9)$.

Lemma 3.4. Let $a_{j-1}, a_{j}, \ldots, a_{n+1}$ be real numbers and $\beta=a_{n+1}-\left\lfloor a_{n+1}\right\rfloor$. Assume that $a_{j-1}>1$ and $a_{j} \geq a_{j+1} \geq \cdots \geq a_{n} \geq a_{n+1}>1$. If $\frac{a_{n}}{a_{n+1}} \beta<1$, and

$$
\prod_{i=j}^{n+1}\left(a_{i}-1\right)>(n+1) \sum_{k=1}^{\left\lfloor a_{n+1}\right\rfloor-1}\left[\frac{(k+\beta)^{j-1}}{a_{n+1}^{j-1}} \prod_{i=j}^{n}\left(\frac{a_{i}}{a_{n+1}}(k+\beta)-1\right)\right]
$$

then

$$
\prod_{i=j-1}^{n+1}\left(a_{i}-1\right)>(n+1) \sum_{k=1}^{\left\lfloor a_{n+1}\right\rfloor-1}\left[\frac{(k+\beta)^{j-2}}{a_{n+1}^{j-2}} \prod_{i=j-1}^{n}\left(\frac{a_{i}}{a_{n+1}}(k+\beta)-1\right)\right] .
$$

Proof. The proof of Lemma 3.4 is similar to that of Lemma 3.3. For fixed $a_{j} \geq a_{j+1} \geq \cdots \geq a_{n} \geq a_{n+1}>1$, let

$$
G\left(a_{j-1}\right)=\prod_{i=j-1}^{n+1}\left(a_{i}-1\right)-(n+1) \sum_{k=1}^{\left\lfloor a_{n+1}\right\rfloor-1}\left[\frac{(k+\beta)^{j-2}}{a_{n+1}^{j-2}} \prod_{i=j-1}^{n}\left(\frac{a_{i}}{a_{n+1}}(k+\beta)-1\right)\right] .
$$

It suffices to show that $G\left(a_{j-1}\right)$ is a strictly increasing function of $a_{j-1}$ and $G(1) \geq 0$.

Letting $a_{j-1}=1$, it can be seen that $\frac{a_{j-1}}{a_{n+1}}(k+\beta)-1<0$ for $1 \leq k \leq\left\lfloor a_{n+1}\right\rfloor-1$. Furthermore, since $k \geq 1$ and $a_{j} \geq a_{j+1} \geq \cdots \geq a_{n} \geq a_{n+1}$, we have

$$
\prod_{i=j}^{n}\left(\frac{a_{i}}{a_{n+1}}(k+\beta)-1\right) \geq 0 \text { for } 1 \leq k \leq\left\lfloor a_{n+1}\right\rfloor-1,
$$

and

$$
(n+1) \sum_{k=1}^{\left\lfloor a_{n+1}\right\rfloor-1}\left[\frac{(k+\beta)^{j-2}}{a_{n+1}^{j-2}} \prod_{i=j-1}^{n}\left(\frac{a_{i}}{a_{n+1}}(k+\beta)-1\right)\right] \leq 0 .
$$

Therefore, $G(1) \geq 0$. We then compute

$$
\begin{aligned}
\frac{d G}{d a_{j-1}} & =\prod_{i=j}^{n+1}\left(a_{i}-1\right)-(n+1) \sum_{k=1}^{\left\lfloor a_{n+1}\right\rfloor-1}\left[\frac{(k+\beta)^{j-1}}{a_{n+1}^{j-1}} \prod_{i=j}^{n}\left(\frac{a_{i}}{a_{n+1}}(k+\beta)-1\right)\right] \\
& >0 \text { by (3.14). }
\end{aligned}
$$

so $G\left(a_{j-1}\right)$ is a strictly increasing function of $a_{j-1}$. We conclude that $G\left(a_{j-1}\right)>0$ for $a_{j-1}>1$. 


\section{Main result}

The purpose of this section is to prove Theorem 1.1 (the Rough Estimate GLY conjecture).

We first consider the case where $a_{n}>1$. Our intention is to show that if Theorem 1.1 is true for $n$-dimensional simplices, then it must also be true for $(n+1)$-dimensional simplices. From $[\mathrm{Xu}-\mathrm{Ya} 1]$, Theorem 1.1 was proven for $n=3$, which shall be our base case in the induction. Let $n \geq 3$ and $P_{n}=P_{\left(a_{1}, a_{2}, \ldots, a_{n}\right)}$ be the number of positive integral solutions satisfying

$$
\frac{x_{1}}{a_{1}}+\frac{x_{2}}{a_{2}}+\cdots+\frac{x_{n}}{a_{n}} \leq 1,
$$

where $a_{1}, a_{2}, \ldots, a_{n}$ are positive real numbers such that $a_{1} \geq a_{2} \geq \cdots \geq a_{n}>1$. By the induction hypothesis, we have

$$
n ! \cdot P_{n}<\prod_{i=1}^{n}\left(a_{i}-1\right) .
$$

Consider $P_{n+1}=P_{\left(a_{1}, a_{2}, \ldots, a_{n+1}\right)}$, which is the number of positive integral solutions satisfying

$$
\frac{x_{1}}{a_{1}}+\frac{x_{2}}{a_{2}}+\cdots+\frac{x_{n+1}}{a_{n+1}} \leq 1,
$$

where $a_{1}, a_{2}, \ldots, a_{n+1}$ are positive real numbers such that $a_{1} \geq a_{2} \geq \cdots \geq a_{n+1}>1$. We slice the $(n+1)$-dimensional simplex described by (4.3) along the $x_{n+1}$ axis into $\left\lfloor a_{n+1}\right\rfloor$ similar $n$-dimensional simplices described by (4.1). Specifically, the $n$ dimensional simplex at $x_{n+1}=\left\lfloor a_{n+1}\right\rfloor-k$, where $\left\lfloor a_{n+1}-1\right\rfloor \geq k \geq 0$, is

$$
\frac{x_{1}}{\frac{a_{1}}{a_{n+1}}(k+\beta)}+\frac{x_{2}}{\frac{a_{2}}{a_{n+1}}(k+\beta)}+\cdots+\frac{x_{n}}{\frac{a_{n}}{a_{n+1}}(k+\beta)} \leq 1,
$$

where $\beta=a_{n+1}-\left\lfloor a_{n+1}\right\rfloor$. We are going to consider two cases.

Case 1: $\frac{a_{n}}{a_{n+1}} \beta \geq 1$

When we sum up the number of lattice points in each $n$-dimensional simplex described by (4.4), we have the following estimate according to the induction hypothesis in (4.2).

$$
n ! \cdot P_{n+1}<\sum_{k=0}^{\left\lfloor a_{n+1}\right\rfloor-1}\left[\prod_{i=1}^{n}\left(\frac{a_{i}}{a_{n+1}}(k+\beta)-1\right)\right] .
$$

Our goal in this case is to show that

$$
(n+1) \sum_{k=0}^{\left\lfloor a_{n+1}\right\rfloor-1}\left[\prod_{i=1}^{n}\left(\frac{a_{i}}{a_{n+1}}(k+\beta)-1\right)\right]<\prod_{i=1}^{n+1}\left(a_{i}-1\right) .
$$

From Proposition 3.1, we have

$$
(n+1) \sum_{k=0}^{\left\lfloor a_{n+1}\right\rfloor-1} \frac{(k+\beta)^{n}}{a_{n+1}^{n}}<\left(a_{n+1}-1\right) .
$$


If we repeatedly apply Lemma 3.3 to (4.7), then after $n$ times we will have (4.6). The inequality sign in (4.6) indicates that when $a_{n+1}>1$, Theorem 1.1 is strictly larger than $P_{n+1}$.

Case 2: $\frac{a_{n}}{a_{n+1}} \beta<1$

Examining (4.4) closely, we see that if $\frac{a_{n}}{a_{n+1}} \beta<1$, then the number of lattice points is zero in the layer $k=0$ of the $(n+1)$-dimensional simplex. Therefore, $k$ goes from 1 to $\left\lfloor a_{n+1}\right\rfloor-1$ in this case.

From (4.2), the number of lattice points in (4.3) has the following estimate:

$$
n ! \cdot P_{n+1}<\sum_{k=1}^{\left\lfloor a_{n+1}\right\rfloor-1}\left[\prod_{i=1}^{n}\left(\frac{a_{i}}{a_{n+1}}(k+\beta)-1\right)\right] .
$$

Therefore we only need to show that if $\frac{a_{n}}{a_{n+1}} \beta<1$, then

$$
(n+1) \sum_{k=1}^{\left\lfloor a_{n+1}\right\rfloor-1}\left[\prod_{i=1}^{n}\left(\frac{a_{i}}{a_{n+1}}(k+\beta)-1\right)\right]<\prod_{i=1}^{n+1}\left(a_{i}-1\right) .
$$

From Proposition 3.1, we have

$$
\begin{aligned}
\left(a_{n+1}-1\right) & >(n+1) \sum_{k=0}^{\left\lfloor a_{n+1}\right\rfloor-1} \frac{(k+\beta)^{n}}{a_{n+1}^{n}} \\
& >(n+1) \sum_{k=1}^{\left\lfloor a_{n+1}\right\rfloor-1} \frac{(k+\beta)^{n}}{a_{n+1}^{n}} .
\end{aligned}
$$

Again, we can repeatedly apply Lemma 3.4 to (4.10), and after $n$ times we will have (4.9). Thus far, we have shown that if $a_{1} \geq a_{2} \geq a_{3} \geq \cdots \geq a_{n}>1$, then $n ! \cdot P_{n}<\left(a_{1}-1\right)\left(a_{2}-1\right) \cdots\left(a_{n}-1\right)$. Finally, notice that if $a_{n}=1$, then the number of lattice points in any $n$-dimensional simplex becomes zero, so our upper estimate becomes an equality if and only if $a_{n}=1$. This completes the proof of Theorem 1.1 .

\section{Acknowledgments}

We thank the referees for providing useful comments for improving the presentation of our paper.

\section{References}

[Ba] A. I. Barvinok, Computing the Ehrhart polynomial of a convex lattice polytope, Discrete Comput. Geom. 12 (1994) 35-48.

[Ba-Po] A. I. Barvinok and J. Pommersheim, An algorithmic theory of lattice points in polyhedra, New Perspectives in Mathematics, MSRI, 38 (1999) 91-147.

[Br-Ve] M. Brion and M. Vergne, Lattice points in simple polytopes, J. Amer. Math. Soc. 10 (1997) 371-392.

[Ca-Sh] S. E. Cappell and J. L. Shaneson, Genera of algebraic varieties and counting of lattice points, Bull. Amer. Math. Soc. (N.S.) 30 (1994), no. 1, 62-69.

[Co] L. Comtet, Advanced Combinatorics, Dr. Reided Publishing Company, 1997. 
[Di-Ro] R. Diaz and S. Robins, The Ehrhart polynomial of a lattice polytope, Ann. of Math. 145 (1997) 503-518.

[Du] A. Durfee, The signature of smoothings of complex surface singularities, Ann. of Math. 232 (1978) 85-98.

[Gr] A. Granville, letter to Y.-J. Xu, 1992.

[Ha-Li 1] G. H. Hardy and J. E. Littlewood, Some Problems of Diophantine Approximation, in "Proc. 5th Int. Congress of Mathematics" (1912), 223-229.

[Ha-Li 2] _ The lattice points of a right-angled triangle, Proc. London Math. Soc. (2) 20 (1921) 15-36.

[Ha-Li 3] $\ldots$, The lattice points of a right-angled triangle (second memoir), Hamburg Math. Abh. 1 (1922) 212-249.

[Ka-Kh] J. M. Kantor and A.Khovanskii, Une application du Theoreme de Riemann-Roch combinatoire au polynome d'Ehrhart des polytopes entier de $\mathbb{R}^{d}$, C. R. Acad. Sci. Paris, Series I 317 (1993) 501-507.

[Li-Ya 1] K.-P. Lin and S. S.-T. Yau, Analysis of Sharp Polynomial Upper Estimate of Number of Positive Integral Points in 4-dimensional Tetrahedra, J. Reine Angew. Math. 547 (2002), 191-205.

[Li-Ya 2] _ A sharp upper estimate of the number of integral points in 5-dimensional tetrahedra, J. Number Theory 93 (2002) 207-234.

[Li-Ya 3] Counting the number of integral points in general $n$-dimensional tetrahedra and Bernoulli polynomials, Canad. Math. Bull. 24 (2003) 229-241.

[Li-Ya 4] _ Classification of affine varieties being cones over nonsingular projective varieties: hypersurface case, Communication in Analysis and Geometry, Vol. 12 (2004), no. 5, 12011219.

[Me-Te] M. Merle and B. Teissier, Conditions d'Adjonction d'Après du val, seminaire sur les singularités des surfaces, Springer Lect. Notes Math. 777, (1980)229-245.

[Mi-Or] J. Milnor and P. Orlik, Isolated singularities defined by weighted homogeneous polynomials, Topology 9 (1970) 385-393.

[Mo] L. J. Mordell, Lattice points in a tetrahedron and generalized Dedekind sums, J. Indian Math. 15 (1951) 41-46.

[Po] J. E. Pommersheim, Toric varieties, lattice points, and Dedekind sums, Math. Annalen 295 (1993) 1-24.

[Pom 1] C. Pomerance, The role of smooth numbers in Number Theoretic Algorithm, ICM Zürich (1994) 411-422.

[Pom 2] Lecture Notes on Primality Testing and Factoring: A Short Course, Kent State University, 1984.

[Sa] K. Saito, Quasihomogene isolierte singularitäten von hyperflächen, Invent. Math. 14 (1971) 123-142.

[Sp 1] D. C. Spencer, On a Hardy-Littlewood problem of diophantine approximation, Math. Proc. Cambridge Philos. Soc. 35 (1939) 527-547.

[Sp 2] - The lattice points of tetrahedra, J. Math. Phys. 21 (1942)189-197.

[Wa-Ya] X. Wang and S. S.-T. Yau, On the GLY Conjecture of upper estimate of positive integral points in real tetrahedra, preprint.

[Xu-Ya 1] Y.-J. Xu and S. S.-T. Yau, A sharp estimate of the number of integral points in a tetrahedron, J. Reine Angew. Math. 423 (1992) 199-219.

[Xu-Ya 2] S Sharp estimate of the number of integral points In a 4-dimensional tetrahedron, J. Reine Angew. Math. 473 (1996) 1-23.

Department of Mathematics, Statistics \& Computer Science, M/C 249, University of Illinois at Chicago, 851 S. Morgan St., Chicago, Illinois, 60607

E-mail address: yau@uic.edu

Illinois Mathematics and Science Academy, 1500 W. Sullivan Rd., Aurora, Illinois, 60506

E-mail address: letian@imsa.edu 